\title{
Desempenho agronômico de genótipos de amendoim tipo runner, visando colheita antecipada
}

Programa de Melhoramento do Amendoim - Embrapa, Santo Antônio de Goiás, GO, kennedybrunno22@gmail.com.

Programa de Melhoramento do Amendoim - Embrapa, Santo Antônio de Goiás, GO, jair.heuert@embrapa.br.

\section{Lúcio Lima Rodrigues ${ }^{\mathbb{D}}$}

Discente de Engenharia Básica da UNIP, Goiânia, GO, luciolimarodrigues@gmail.com.

Discente de Agronomia do IFMT Campus São Vicente - Centro de Referência de Campo Verde, Campo Verde, MT, maxuelfellipe90@gmail.com.

Taís de Moraes Falleiro Suassuna (iD Programa de Melhoramento do Amendoim - Embrapa, Santo Antônio de Goiás, GO, tais.suassuna@embrapa.br.

\section{RESUMO}

Genótipos de amendoim do tipo runner precoces são determinantes para o cultivo do amendoim em áreas de renovação de cana-de-açúcar. $O$ ensaio foi instalado na área experimental da Embrapa Arroz e Feijão em Santo Antônio de Goiás-GO. 0 delineamento empregado foi em blocos casualizados, contendo quatro repetições. Os tratamentos constaram de sete linhagens (18-2173 OL, 18-2250 OL, 18-2133 OL, 18-2101 OL, 18-2266 OL, 17-1253 OL e 18-2094 OL) e uma cultivar (BRS $423 \mathrm{OL}$ ). A área útil de cada parcela era de $5,4 \mathrm{~m}^{2}$, constituídas por duas linhas de três metros de comprimento, com espaçamento de 0,90 m. As plantas foram invertidas aos 118 dias após a emergência (DAE). Avaliou-se porcentagem de grãos maduros, severidade de mancha preta, massa de 100 grãos e produtividade, observando diferença significativa para todas as variáveis. Todas as linhagens apresentaram maturação dos grãos acima de $70 \%$. As menores notas de severidade de mancha preta foram obtidas nos genótipos 18-2173 OL, 18-2094 OL, BRS 423 OL, 18-2133 OL, 18-2266 OL, 18-2250 OL e 17-1253 OL. Os

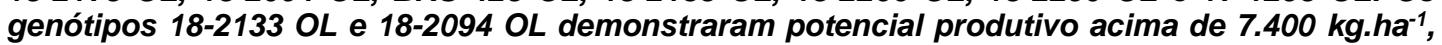
quando colhidos antes dos 120 DAE.

Palavras-chave: Arachis hypogaea L.; Programa de Melhoramento; Precocidade.

\section{Agronomic performance of runner peanut genotypes, aiming for early harvest}

\section{ABSTRACT}

Early runner type peanut genotypes are crucial for growing peanuts in sugarcane renewal areas. The test was installed in the experimental area of Embrapa Arroz e Feijão in Santo Antônio de Goiás-GO. The design used was in randomized blocks, containing four replications. The treatments consisted of seven strains (18-2173 OL, 18-2250 OL, 18-2133 OL, 18-2101 OL, 18$2266 O L, 17-1253 \mathrm{OL}$ and 18-2094 OL) and one cultivar (BRS $423 \mathrm{OL}$ ). The useful area of each plot was $5.4 \mathrm{~m}^{2}$, consisting of two lines three meters long, with a spacing of $0.90 \mathrm{~m}$. The plants were inverted at 118 days after emergence (DAE). Percentage of mature grains, severity of black 
spot, mass of 100 grains and yield were evaluated, observing significant difference for all variables. All strains showed grain maturity above $70 \%$. The lowest grades of black spot severity were obtained from genotypes 18-2173 OL, 18-2094 OL, BRS 423 OL, 18-2133 OL, 18$2266 O L, 18-2250 \mathrm{OL}$ and 17-1253 OL. The genotypes 18-2133 OL and 18-2094 OL showed yield potential above 7,400 kg.ha-1, when harvested before 120 DAE.

Keywords: Arachis hypogaea L; Improvement Program; Precocity.

\title{
Rendimiento agronómico de los genotipos de maní runner, con el objetivo de una cosecha temprana
}

\section{RESUMEN}

\begin{abstract}
Los genotipos de maní de los primeros corredores son cruciales para el cultivo de maní en las áreas de renovación de la caña de azúcar. La prueba se instaló en el área experimental de Embrapa Arroz e Feijão en Santo Antônio de Goiás-GO. El diseño utilizado fue en bloques aleatorizados, que contienen cuatro repeticiones. Los tratamientos consistieron en siete cepas (18-2173 OL, 18-2250 OL, 18-2133 OL, 18-2101 OL, 18-2266 OL, 17-1253 OL y 18-2094 OL) y un cultivar (BRS $423 \mathrm{OL}$ ). El área útil de cada parcela fue de $5.4 \mathrm{~m}^{2}$, que consta de dos líneas de tres metros de largo, con una separación de $0.90 \mathrm{~m}$. Las plantas se invirtieron a los 118 días después de la emergencia (DAE). Se evaluó el porcentaje de granos maduros, la gravedad de la mancha negra, la masa de 100 granos y el rendimiento, observando diferencias significativas para todas las variables. Todas las cepas mostraron madurez de grano superior al $70 \%$. Los grados más bajos de severidad de la mancha negra se obtuvieron de los genotipos 18-2173 OL, 18-2094 OL, BRS 423 OL, 18-2133 OL, 18-2266 OL, 18-2250 OL y 17-1253 OL. Los genotipos 18-

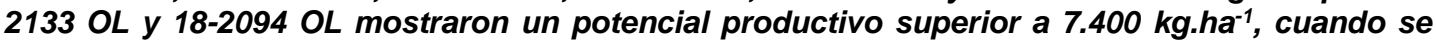
cosecharon antes de 120 DAE.
\end{abstract}

Palabras clave: Arachis hypogaea L.; Programa de mejoramiento; Precocidad.

\section{Introdução}

O cultivo do amendoim ocorre predominantemente em áreas de renovação de cana-de-açúcar, um sistema de sucessão de culturas importante para a diversificação da exploração agrícola (CONAB, 2020). Neste contexto, necessita-se de cultivares de amendoim precoces, para se ajustar a um período curto que a área fica disponível para renovação, até que ocorra um novo plantio de cana (BETIOL et al., 2019).

No âmbito da identificação de cultivares precoces de amendoim, a avaliação do grau de maturidade das vagens e dos grãos é um componente determinante do ciclo de uma cultivar, feita visualmente conforme o grau de enchimento de grãos, com finalidade de seleção conforme a precocidade da maturação (RUCKER et al., 1994).

O Programa de Melhoramento do Amendoim da Embrapa, trabalha em busca de cultivares adaptadas as regiões promissoras de expansão do 
amendoim no país, visando obter materiais com precocidade, padrão runner (granulometria e forma das vagens e grãos), alto teor de ácido oleico (>70\%) e maior produtividade. Assim, são realizados ensaios testando diferentes linhagens em comparação as cultivares do mercado, analisando estes fatores primordiais na seleção (COSTA et al., 2016; MARTINS et al., 2018).

O presente trabalho teve como objetivo avaliar a porcentagem de grãos maduros, severidade de mancha preta, massa de 100 grãos e produtividade em oito genótipos de amendoim.

\section{Material e métodos}

O experimento foi realizado na área experimental da Embrapa Arroz e Feijão, no ano agrícola 2019/20, com semeadura manual realizada no dia 28 de novembro de 2019. Foi utilizado delineamento em blocos casualizados, com quatro repetições. Os tratamentos constaram de oito genótipos, sendo sete linhagens (18-2173 OL, 18-2250 OL, 18-2133 OL, 182101 OL, 18-2266 OL, 17-1253 OL e 18-2094 OL) e uma cultivar (BRS 423 $\mathrm{OL}$ ), todas desenvolvidas pelo Programa de Melhoramento do Amendoim da Embrapa.

As parcelas eram compostas por duas linhas de três metros de comprimento, espaçadas em $0,90 \mathrm{~m}$, com intervalo de dois metros entre parcelas e área útil para avaliação de $5,4 \mathrm{~m}^{2}$ na parcela. Os manejos cultural e fitossanitário foram realizados de acordo com as recomendações para a cultura. $O$ estande médio foi de 15 plantas por metro linear. $O$ ensaio foi invertido no dia 1 de abril de 2020, aos 118 dias após a emergência (DAE).

Foram avaliados grãos maduros (\%), severidade de mancha preta (notas) massa de 100 grãos $(\mathrm{g})$ e produtividade de vagens $\left(\mathrm{kg} \cdot \mathrm{ha}^{-1} \mathrm{e}\right.$ sacas.alqueire $\left.{ }^{-1}\right)$. Os grãos maduros (\%) foram avaliados como descrito por Rucker et al. (1994). A severidade de mancha preta (Cercosporidium personatum) foi avaliada usando a escala diagramática da incidência com notas de 1 a 9 antes da inversão do experimento (SUBRAHMANYAM et al., 1982). Os dados foram submetidos à análise de variância pelo Teste $F e$ as médias dos tratamentos foram comparados pelo teste de Scott-Knott, no 
nível de $5 \%$ de probabilidade, por meio do programa computacional SISVAR 5.6 (FERREIRA, 2019).

\section{Resultados e discussão}

Houve diferença significativa para os diferentes genótipos de amendoim dentre as variáveis grãos maduros (\%), severidade de mancha preta (notas), massa de 100 grãos (g) e produtividade (kg.ha ${ }^{-1}$ ) (Tabela 1). Nos grãos maduros (\%), verifica-se que as linhagens $18-2173$ OL $(83,1 \%)$, 18-2250 OL (82,1\%), 18-2133 OL (79,6\%), 18-2101 OL (73,6\%), 18-2266 OL $(72,2 \%)$ e 18-2094 OL $(71,2 \%)$, apresentaram maturação de grão acima de $70 \%$, quando comparados aos demais genótipos, demonstrando característica de serem linhagens propícias para a colheita antecipada, antes do 120 DAE. Somente a cultivar BRS 423 OL (62,7\%), obteve maturação menor que $70 \%$.

As menores notas de severidade de mancha preta foram obtidas nos genótipos 18-2173 OL (4,0), 18-2094 OL (5,0), BRS 423 OL (5,0), 18-2133 OL $(5,3), 18-2266 \mathrm{OL}(5,3), 18-2250 \mathrm{OL}(5,3)$ e 17-1253 OL $(5,8)$, resultando nos genótipos mais tolerantes a mancha preta. Verificou-se a maior nota de severidade na linhagem 18-2101 OL (7,3). Da mesma forma, Martins et al. (2019) relataram a linhagem $18-2101$ OL $(8,4)$ entre as mais suscetíveis a mancha preta quando comparados a 24 genótipos de amendoim em Santo Antônio de Goiás-GO.

A maior massa de 100 grãos (g) foi obtida com o genótipo 18-2094 OL $(91,0 \mathrm{~g})$ e a menor com o $18-2250 \mathrm{OL}(73,7 \mathrm{~g})$ e $18-2101 \mathrm{OL}(74,4 \mathrm{~g})$, resultando num decréscimo de 19 e 18\%, respectivamente. Os genótipos 18$2266 \mathrm{OL}(81,5 \mathrm{~g}), 18-2173 \mathrm{OL}(81,4 \mathrm{~g}), 18-2133 \mathrm{OL}(79,9 \mathrm{~g}), 17-1253 \mathrm{OL}$ $(79,6 \mathrm{~g})$ e BRS $423 \mathrm{OL}(76,8 \mathrm{~g})$, apresentaram massas intermediárias. Martins et al. (2019) obtiveram massa de 75,9 g com a cultivar BRS $423 \mathrm{OL}$. Por outro lado, Betiol et al. (2019), observaram massa de 79,6 g para a cultivar BRS 423 OL, em Ribeirão Bonito-SP. 
Tabela 1. Grãos maduros (\%), severidade de mancha preta (notas), massa de 100 grãos (g) e produtividade de vagens $\left(\mathrm{kg} \mathrm{ha}^{-1}\right.$ e sacas alqueire-1) em função de diferentes genótipos de amendoim. Santo Antônio de Goiás-GO, 2019/20.

\begin{tabular}{cccccc}
\hline Genótipos & $\begin{array}{c}\text { Grãos } \\
\text { maduros (\%) }\end{array}$ & $\begin{array}{c}\text { Severidade } \\
\text { (notas) }\end{array}$ & $\begin{array}{c}\text { Massa de } \\
\mathbf{1 0 0} \text { grãos (g) }\end{array}$ & \multicolumn{2}{c}{ Produtividade de vagens } \\
(kg ha $\left.^{-1}\right)$ & (sacas alqueire $^{-1}$ ) \\
\hline $18-2173 \mathrm{OL}$ & $83,1 \mathrm{a}$ & $4,0 \mathrm{a}$ & $81,4 \mathrm{~b}$ & $7.006,7 \mathrm{~b}$ & 678,2 \\
$18-2250 \mathrm{OL}$ & $82,1 \mathrm{a}$ & $5,3 \mathrm{a}$ & $73,7 \mathrm{e}$ & $6.304,7 \mathrm{c}$ & 610.3 \\
$18-2133 \mathrm{OL}$ & $79,6 \mathrm{a}$ & $5,3 \mathrm{a}$ & $79,9 \mathrm{c}$ & $7.548,3 \mathrm{a}$ & 730,6 \\
$18-2101 \mathrm{OL}$ & $73,6 \mathrm{~b}$ & $7,3 \mathrm{~b}$ & $74,4 \mathrm{e}$ & $6.408,1 \mathrm{c}$ & 620,3 \\
$18-2266 \mathrm{OL}$ & $72,2 \mathrm{~b}$ & $5,3 \mathrm{a}$ & $81,5 \mathrm{~b}$ & $6,771,4 \mathrm{~b}$ & 655,4 \\
$17-1253 \mathrm{OL}$ & $71,4 \mathrm{~b}$ & $5,8 \mathrm{a}$ & $79,6 \mathrm{c}$ & $6.996,2 \mathrm{~b}$ & 677,2 \\
$18-2094 \mathrm{OL}$ & $71,2 \mathrm{~b}$ & $5,0 \mathrm{a}$ & $91,0 \mathrm{a}$ & $7.458,0 \mathrm{a}$ & 721,9 \\
BRS 423 OL & $62,7 \mathrm{c}$ & $5,0 \mathrm{a}$ & $76,8 \mathrm{~d}$ & $6.889,5 \mathrm{~b}$ & 666,9 \\
\hline Média & 74,5 & 5,3 & 79,8 & $6.877,8$ & 654,3 \\
\hline C.V. (\%) & 3,3 & 15,2 & 1,5 & 5,7 & - \\
\hline Pr>Fc & $>0,0001^{*}$ & $0,0012^{*}$ & $>0,0001^{*}$ & $0,0001^{*}$ & - \\
\hline *- significativo a 5\% de probabilidade pelo teste de Scott-Knott; ns - não significativo; C.V. \\
- coeficiente de variação.
\end{tabular}

Sobre a produtividade de vagens $\left(\mathrm{kg}^{\mathrm{h}} \mathrm{ha}^{-1}\right)$, verifica-se que os genótipos 18-2133 OL (7.548,3 kg.ha-1) e 18-2094 OL (7.458,0 kg.ha-1), foram os mais produtivos do presente estudo, quando comparados aos demais, obtendo produtividades superiores a $7.400 \mathrm{~kg} \mathrm{ha}^{-1}$. Observa-se que os genótipos 18-2173 OL (7.006,7 kg.ha-1 $), 17-1253$ OL (6.996,2 kg.ha-1), BRS $423 \mathrm{OL}\left(6.889,5 \mathrm{~kg} \cdot \mathrm{ha}^{-1}\right)$ e 18-2266 OL (6.771,4 kg.ha-1), obtiveram produtividades intermediárias. Corroborando com Martins et al. (2019), que também obtiveram as maiores produtividades com o genótipo 18-2094 OL (7.343,6 kg.ha-1) e intermediária com o genótipo 17-1253 OL (6.735,6 kg.ha$\left.{ }^{1}\right)$. As menores produtividades foram obtidas nos genótipos 18-2250 OL $\left(6.304,7 \mathrm{~kg} \cdot \mathrm{ha}^{-1}\right)$ e $18-2101 \mathrm{OL}\left(6.408,1 \mathrm{~kg} \cdot \mathrm{ha}^{-1}\right)$.

\section{Conclusões}

Todas as linhagens apresentaram maturação dos grãos acima de $70 \%$. As menores notas de severidade de mancha preta foram obtidas nos genótipos 18-2173 OL, 18-2094 OL, BRS 423 OL, 18-2133 OL, 18-2266 OL, 18-2250 OL e 17-1253 OL. Os genótipos 18-2133 OL e 18-2094 OL 
demonstraram potencial produtivo acima de $7.400 \mathrm{~kg} \mathrm{ha}^{-1}$, quando colhidos antes dos 120 DAE.

\section{Agradecimentos}

Os autores agradecem as empresas que são parceiras do Programa de Melhoramento do Amendoim - Embrapa. Estes resultados estão vinculados ao projeto SEG 20.18.01.021.00. 


\section{Referências}

BETIOL, R. A. B.; HEUERT, J.; MARTINS, K. B. B.; SUASSUNA, T. M. F. Desempenho de cultivares de amendoim tipo runner na região central do estado de São Paulo, visando a colheita antecipada. In: Anais do Encontro Sobre a Cultura do Amendoim, 16., 2019, Jaboticabal. Anais eletrônicos... Campinas: GALOÁ, 2019. Disponível em: $<$ https://proceedings.science/encontro-amendoim-2019/papers/desempenhode-cultivares-de-amendoim-tipo-runner-na-regiao-central-do-estado-de-saopaulo--visando-a-colheita-antecipada>. Acesso em: 21 abr. 2020.

CONAB. Acompanhamento da Safra Brasileira de grãos, v. 7, n. 5 - Safra 2019/20 - Quinto levantamento, Brasília, p. 1-112, fev. 2020. Disponível em: $<$ https://www.conab.gov.br/info-agro/safras/graos>. Acesso em: 20 abr. 2020.

COSTA, L. C.; HEUERT, J.; SUASSUNA, N. D.; SUASSUNA, T. M. F. Desempenho de linhagens avançadas de amendoim selecionadas para cultivo no cerrado brasileiro. In: Seminário Jovens Talentos, 10., 2016, Santo Antônio de Goiás. Resumos... Santo Antônio de Goiás: Embrapa Arroz e Feijão, 2016. Disponível em: <http://ainfo.cnptia.embrapa.br/digital/bitstream/item/157311/1/Desempenhode-linhagens-avancadas.pdf >. Acesso em: 21 abr. 2020.

FERREIRA, D. F. SISVAR: a computer analysis system to fixed effects split plot type designs. Revista Brasileira de Biometria, [S.L.], v. 37, n. 4, p. 529, 20 dez. 2019. http://dx.doi.org/10.28951/rbb.v37i4.450.

MARTINS, K. B. B.; SUASSUNA, T. M. F.; HEUERT, J.; RIBEIRO, R. P.; SOAVE, J. H.; SANTOS, L. C. C. Avaliação em genótipos de amendoim tipo runner visando a colheita antecipada. In: Anais do Encontro Sobre a Cultura do Amendoim, 15., 2018, Jaboticabal. Anais eletrônicos... Campinas: GALOÁ, 2018. Disponível em: <https://proceedings.science/encontroamendoim-2018/papers/avaliacao-em-genotipos-de-amendoim-tipo-runnervisando-a-colheita-antecipada>. Acesso em: 21 abr. 2020.

MARTINS, K. B. B.; RODRIGUES, L. L.; HEUERT, J.; XAVIER, M. F. N.; SUASSUNA, T. M. F.; BETIOL, R. A. B. Desempenho agronômico de novas linhagens de amendoim no cerrado. In: Anais do Encontro Sobre a Cultura do Amendoim, 16., 2019, Jaboticabal. Anais eletrônicos... Campinas: GALOÁ, 2019. Disponível em: <https://proceedings.science/encontroamendoim-2019/papers/desempenho-agronomico-de-novas-linhagens-deamendoim-no-cerrado>. Acesso em: 22 abr. 2020.

RUCKER, K. S.; KVIEN, C. C. K.; VELLIDIS, G.; HILL, N. S.; SHARPE, J. K. $A$ visual method of determining maturity of shelled peanuts. Peanut Science, v.21, p.143-146, 1994. https://doi.org/10.3146/i0095-3679-21-2-16

SUBRAHMANYAM, P.; MCDONALD, D.; GIBBONS, R. W.; NIGAM, S. N.; NEVILL, D. J. Resistance to rust and late leaf spot diseases in some 
genotypes of Arachis hypogaea. Peanut Science, v. 9, n. 1, p. 6-10, 1982. https://doi.org/10.3146/i0095-3679-9-1-2 